\title{
Novel surface speckle preparation method for imaging techniques for clay models
}

\author{
M. G. OTTOLINI* and J. DIJKSTRA†
}

\begin{abstract}
A novel method for the application of contrasting speckle material on a clay sample was developed. In comparison to traditional methods, the new method minimises the sample disturbance resulting from sample handling and the removal of any transparent windows of the strongbox after the consolidation stage. This method applies the contrast material on the transparent window before it is released to the surface of the slurry, thus facilitating tracking of material displacements during the consolidation stage and subsequent loading stages. The method was successfully demonstrated for a centrifuge model test and a $1 \mathbf{g}$ model test, each with complex material deformations and rotations.
\end{abstract}

KEYWORDS: centrifuge modelling; deformation; footings/foundations; model tests

ICE Publishing: all rights reserved

\section{INTRODUCTION}

With the increasing availability of low-cost high-resolution digital cameras and open-source image processing tools, fullfield measurement techniques have become popular for quantifying the kinematic response of a physical model. Two-dimensional (2D) plane observations through a transparent window that capture the evolution of particle movements with digital photography are the most popular (e.g. White et al., 2001, 2003; Hall et al., 2010). The image data are processed to extract kinematic evidence using $2 \mathrm{D}$ or three-dimensional digital image correlation (or particle image velocimetry (PIV)), particle tracking, stereophotogrammetry or image subtraction techniques (Sutton et al., 1983; White et al., 2003; Desrues \& Viggiani, 2004; Ochiai et al., 2006; Hall et al., 2010; Rosenbrand \& Dijkstra, 2012; Dijkstra et al., 2013). These techniques make the application of grids or embedded markers (e.g. Allersma, 1997) obsolete. Typically, in coarse-grained materials, the natural contrast of the grains is usually sufficient for subsequent data processing. However, in fine-grained materials with a low contrast, such as rock and clay samples, an additional speckle pattern needs to be applied on the surface. In general, the subsequent image processing will yield more accurate results if the differences between the minimum and maximum intensity levels resulting from the contrast of the speckles are maximised, and the spatial accuracy will improve for dense speckle patterns (Badulescu et al., 2010). On plane stress rock samples, the procedure followed in experimental mechanics (i.e. spray painting the surface) can be followed without significantly influencing the specimen (Hall, 2012). However, clay samples are much more sensitive to additives in the material than are rock samples, and samples are typically reconstituted starting from a clay slurry in a plane strain box.

Common speckles used on clay surfaces are dyed sand or plastic flock particles (White et al., 2003; O'Loughlin et al.,

Manuscript received 4 November 2013; first decision 10 February 2014; accepted 13 March 2014.

Published online at www.geotechniqueletters.com on 10 April 2014.

${ }^{*}$ Geo-Engineering Section, Delft University of Technology, Delft, The Netherlands

$\dagger$ Department of Civil and Environmental Engineering, Chalmers University of Technology, Gothenburg, Sweden
2006, Dingle et al., 2008; Leung et al., 2008; Hossain \& Randolph, 2010). Speckling of these materials onto the clay surface is performed after the consolidation stage. This requires access to the clay sample (i.e. by removing the transparent window). Most authors do not clearly report on this necessary step and its possible impact on the subsequent soil response. Only Hossain \& Randolph (2010) reported removal of the window explicitly, without discussing its effects. The process results in unloading of the sample, resulting in potential contraction/swelling of the sample in the out-of-plane direction and potential changes in the water content of the sample after reconsolidation and the soil-surface contact. Alternatively, the contrast material can be mixed in slurry, which potentially alters the material properties, especially when attempting to reconstitute structured clay (Meijer \& Dijkstra, 2013). However, these techniques are typically used for transparent soils (e.g. Ni et al., 2010) where the additional information gained from the test offsets any deviations in the soil response resulting from the embedded markers or the soil substitute.

As a result, both methods have some limitations, especially for soft clays. A novel approach is proposed here, in which the contrast material is applied to the clay surface without removing the window of the strongbox, thus avoiding additional disturbances after consolidation of the sample. These disturbances are shown to be reasonably small when the research aims to study penetration resistance in stiff clays only. Any additional change in water content, which results from the reconsolidation of the sample, and subsequent change in undrained shear strength is small. By means of penetration resistance of half and full spudcans, Xie (2009) showed that this is the case. However, this is not sufficient when the (anisotropic) stiffness and strength evolution in soft clays adjacent to an embedded foundation element are of concern. Research into sample disturbance resulting from retrieving field samples has shown that the stress-strain response at small strain levels and compressibility parameters are especially influenced (Lunne et al., 2006).

\section{SPECKLE APPLICATION METHODOLOGY}

In the proposed method lightweight plastic particles are used as speckle material, because of their low weight, large 
range of available colours and ease of application. The novelty is that these are first applied to the transparent window before being released to the clay slurry. In this process the particles will not significantly move from the projected position (e.g. float to the surface level or penetrate into the slurry). The advantage of this method is that the window does not need to be released from the strongbox, as would be the case in the approaches where speckles are applied to the clay surface after consolidation.

The challenge was in finding a suitable agent to treat the transparent window that, combined with the lightweight particles, would preserve the speckle before releasing it to the slurry surface. Previous attempts with heavier speckle material (sand grains) and a poor release agent led to sagging of the pattern at elevated stress levels. Best results were achieved by first spraying the transparent window with polytetrafluoroethylene (PTFE) spray (an oil with PTFE particles). The spray is easy to apply, reduces wall friction and holds the particles on the window surface before the slurry is poured. Particles are gently blown onto the PTFE film from an overhead transparency while advancing from the back to the top of the strongbox window. Alternatively, a sieve can be used to apply the speckle material, although in this particular case the first method proved to be more convenient for a small strongbox. The window is best oriented downwards on a flat surface. The plastic speckle particles stick to the PTFE film due to surface tension. When the slurry is poured into the strongbox, the particles are pressed into the clay leaving a small layer of clay mixed with contrast material. A conceptual sketch of the necessary steps for the application of speckles is shown in Fig. 1. After preparation of the strongbox with slurry and speckles, the sample is ready for consolidation (e.g. in a geotechnical centrifuge). Soil deformations during consolidation of the clay slurry densify the speckle pattern.

\section{TEST SETUP, SAMPLE PREPARATION AND TEST PROGRAMME}

Test setup

Benchmark tests were carried out in the geotechnical centrifuge at Delft University of Technology. This is a small beam centrifuge with a radius of $1.22 \mathrm{~m}$ (Allersma, 1994) that was recently re-equipped with modern data acquisition and camera facilities. A flight computer with wireless link, data acquisition and motor control are all hosted on the central beam of the centrifuge. An IDS UI6280RE network camera with a resolution of $2560 \times 1920$ pixel $^{2}$ was mounted on one of the centrifuge swings and was internally connected to the flight computer. This camera is equipped with a $2 / 3^{\prime \prime}(\approx 17 \mathrm{~mm}) 5$ megapixel monochrome CCD sensor with a 12-bit depth output. An LED strip was used as a constant light source. A footing and ball probe were jacked with a standard two-axis actuator. This actuator allows for a $110 \mathrm{~mm}$ vertical stroke up to a load of $3 \mathrm{kN}$ and a rate of $1 \mathrm{~mm} / \mathrm{s}$.

\section{Sample preparation}

Kaolin clay was used in the two example laboratory tests. The clay powder was mixed with de-aired water into a slurry with a high water content (more than ten times the liquid limit). The strongbox used in the experiment had transparent Plexiglas windows on the front and back of the box with inner dimensions $(L \times W \times H)$ of $180 \times 155 \times 150 \mathrm{~mm}^{3}$. Before the slurry was poured into the strongbox, all the walls were sprayed with PTFE spray to reduce wall friction. One of the transparent windows was

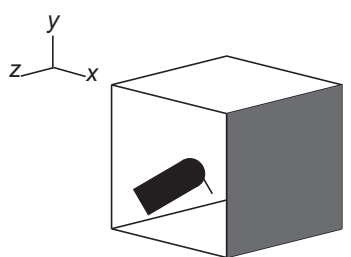

(a)

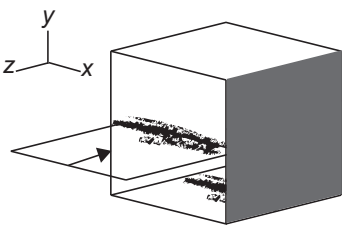

(c)

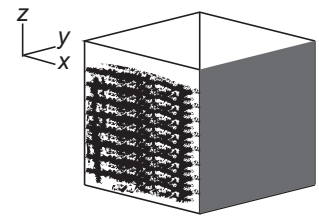

(e)

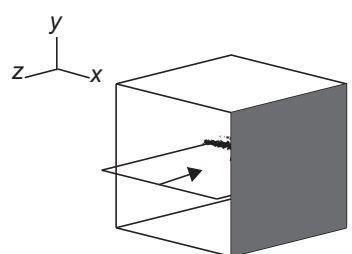

(b)

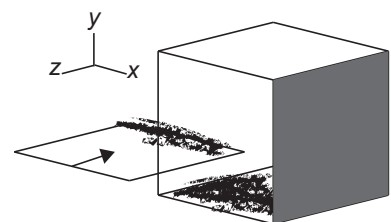

(d)

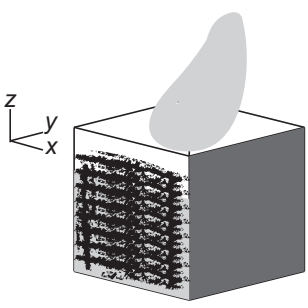

(f)
Fig. 1. Speckle application procedure: (a) spray Perspex plate with PTFE oil; (b), (c), (d) apply contrast material by gently blowing it from a transparency or using a sieve; (e) rotate strongbox vertical; (f) add slurry

subsequently speckled following the method described earlier. Subsequently, the slurry was poured into the strongbox. The clay was consolidated in the TU Delft consolidation centrifuge at $100 \mathrm{~g}$. This centrifuge is a small double swing beam centrifuge of $0.6 \mathrm{~m}$ radius that can be run autonomously for long periods of time at $100 \mathrm{~g}$. This produced a normally consolidated clay sample with an undrained shear strength gradient of $73 \mathrm{kPa} / \mathrm{m}$ at model scale and a water content (throughout the sample height) between 56 and 51\%. Unloading after consolidation effectively results in over-consolidated clay, and the sample was subsequently used for physical model tests at $1 \boldsymbol{g}$ conditions. When the larger centrifuge, equipped with the necessary actuators and data acquisition was also used for the consolidation stage, the procedure of speckling, consolidation and testing could be performed without unloading the sample. However, this is at the expense of effective use of strongbox height as topping up of the sample would require new speckle application. This limitation is only for continuous clay profiles - tests with sand overlaying clay (e.g. embankment on soft soil) do not have this problem.

\section{Test programme}

The first case shows the feasibility of the method for a plane strain penetration test with moderate deformation and rotation at elevated stress levels in a geotechnical centrifuge. A footing of $D_{\mathrm{f}}=20 \mathrm{~mm}$ width and $10 \mathrm{~mm}$ height was penetrated into the clay at $50 \mathrm{~g}$ acceleration in the large TU Delft centrifuge. A foam seal was applied between the footing and the transparent window to ensure a proper failure mechanism around the footing and maintain visibility of the footing during penetration. The test was stopped after $10 \mathrm{~mm}$ penetration $\left(0 \cdot 5 D_{\mathrm{f}}\right)$ at a constant rate of $0 \cdot 1 \mathrm{~mm} / \mathrm{s}$. The image acquisition rate was 4 frames per second (fps). 


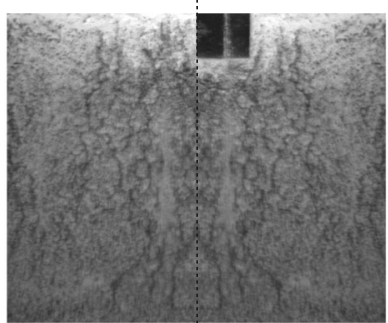

Before test After test

(a)

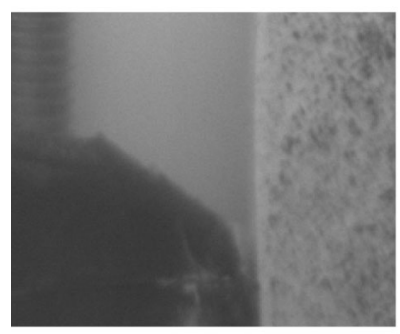

(c)

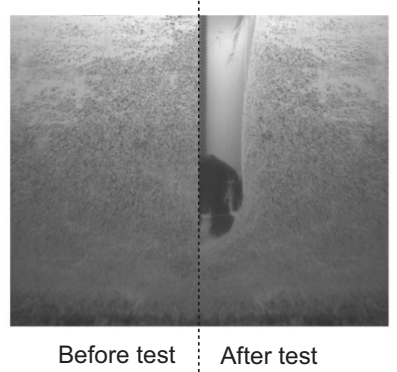

(b)

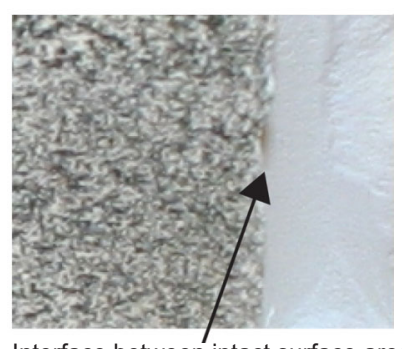

Interface between intact surface area (left) and area where top $0.5 \mathrm{~mm}$ is removed (right)

(d)
Fig. 2. The speckle pattern on kaolin clay after preparation and consolidation (left) and after installation of the probe (right) for (a) footing and (b) ball probe. Close up of ball probe penetration (c) and surface adherence of speckle material on dried sample (d)

The second case investigated the speckle pattern obtained at large deformations, including some out-ofplane deformations. For this purpose, a half symmetric ball probe was penetrated into the clay at $1 \mathrm{~g}$. Again, a constant penetration rate of $0.1 \mathrm{~mm} / \mathrm{s}$ was applied and the maximum applied displacement was $90 \mathrm{~mm}$ for this test. The ball diameter $\left(D_{\mathrm{b}}\right)$ was $30 \mathrm{~mm}$ and the window-ball contact was sealed with a piece of foam. The image acquisition rate was also 4 fps.

\section{THE SPECKLE PATTERN \\ Visual observations}

Figures 2(a) and 2(b) show the speckle pattern after sample preparation and consolidation in the consolidation centrifuge (left-hand images) and after the probes reached the maximum penetration depth (right-hand images) for the $N-\boldsymbol{g}$ footing test (Fig. 2(a)) and the $1 \boldsymbol{g}$ ball probe test (Fig. 2(b)). A high initial speckle density was obtained after preparation and the speckle pattern maintained its contrast even after large material deformations. The difference between the speckle patterns is due to more PTFE in Fig. 2(a) than in Fig. 2(b), resulting in a different distribution. The flocks did not float towards the surface or move, independently from soil movements, into the cavity opened up by the footing or ball penetration (Fig. 2(c)). Further visual inspection of these patterns indicates that only the top surface was somewhat disturbed. This is due to transportation of the strongbox containing slurry, which wipes the window clean of speckles. This can thus easily be avoided by improving sample handling and transport, by removing the top layer (although this partly mitigates the advantages of the method) or by pouring the slurry when the box is installed on the centrifuge swing or in flight (i.e. the standard procedure when testing in a drum centrifuge).

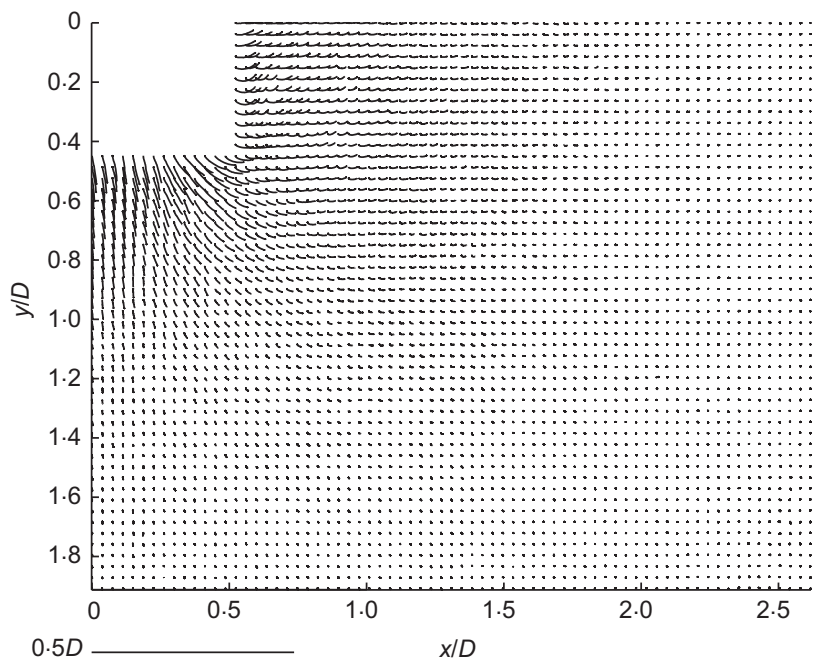

(a)

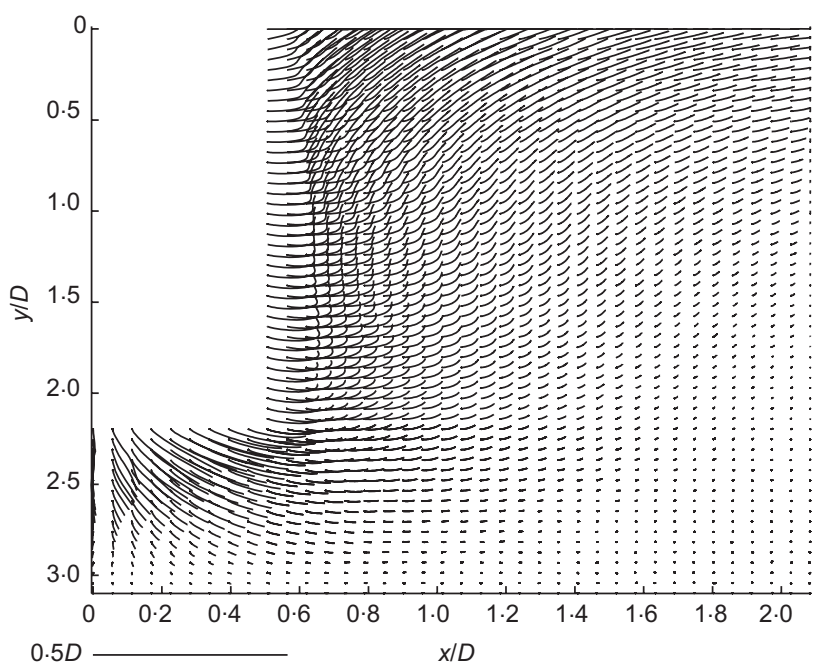

(b)

Fig. 3. Displacement paths extracted from image data of 78 subsequent images for (a) footing and (b) ball probe

Figure 2(d) shows the ball test sample after drying for 1 week at room temperature. Inspection of the dried sample showed that the contrast material penetrated less than $1 \mathrm{~mm}$ depth (measured using a $0.5 \mathrm{~mm}$ scale rule) in the clay and is released from the window. This shows that the contrast material provides a good indication of the deformation in the clay near the transparent window. As well as that, flocks will not significantly alter the material behaviour.

\section{Extracted soil displacement paths}

Similarly to the work of Dijkstra et al. (2013), displacement paths were extracted from the raw image data. These plots contain both translation and rotation information extracted from every displacement increment. First, the displacement fields were extracted from the image sequence using the open-source PIV toolbox JPIV (Vennemann, 2008). The dense speckle pattern with high contrast allowed reduction of the interrogation window size to $8 \times 8$ pixel $^{2}$ on a regular grid of $\mathrm{d} x=\mathrm{d} y=8$ pixels in the last pass of the multi-pass PIV analysis without any spurious vectors. This yielded a spatial resolution of $250 \mu \mathrm{m}$, an accuracy of $30 \mu \mathrm{m}$ and a precision of $30 \mu \mathrm{m}$. The displacement field data were further processed to extract the displacement paths, as shown in Fig. 3, using Matlab. A more objective 


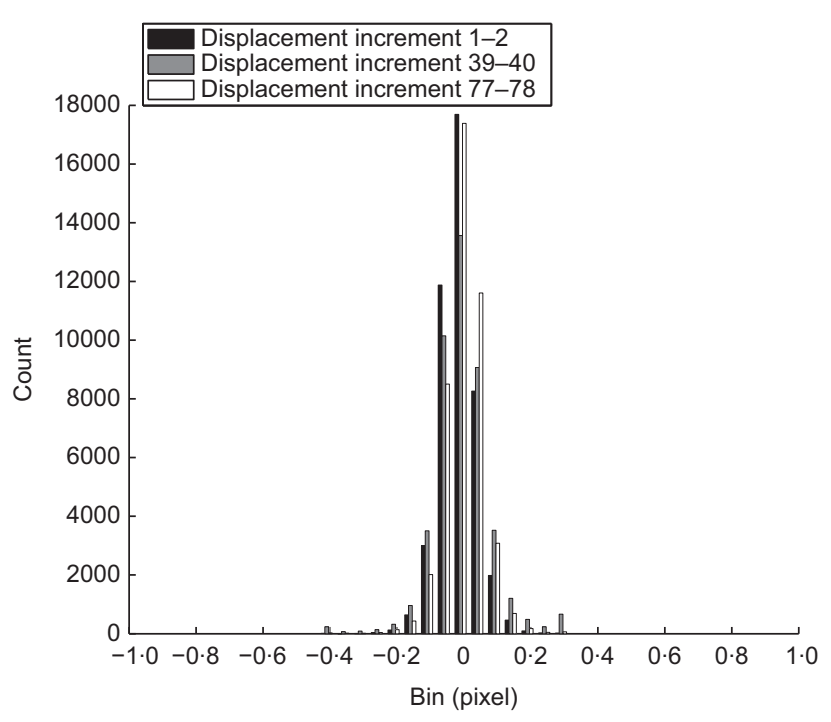

(a)

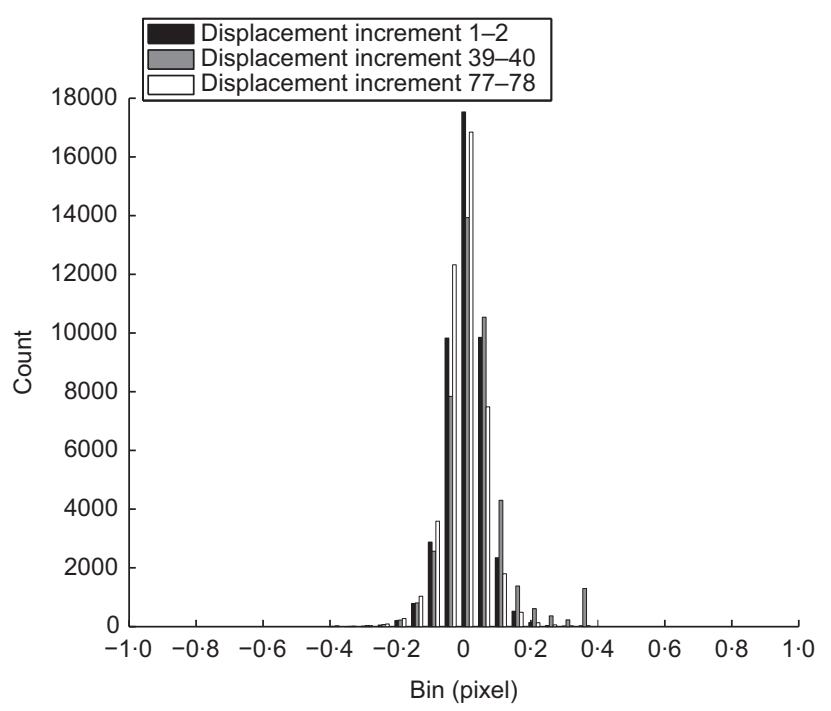

(b)

Fig. 4. Histogram of magnitudes of (a) horizontal and (b) vertical incremental displacement components between images 1 and 2, 39 and 40 and images 77 and 78

measure of the performance is shown by means of the histograms for the horizontal and vertical displacement components plotted in Figs. 4(a) and 4(b), respectively. Three data series, each representing a displacement increment, are shown in each figure. A bin size of 0.05 pixel was used for plotting the data. The figures show that the incremental displacement field from cross-correlating image 1 and image 2 with the original speckle pattern retrieves a similar displacement magnitude distribution to the other displacement increments half way (increment 3940) and at the end of the test (78-79). No integer locking was observed at any stage of the test - this indicates the stability of the speckle pattern and the suitability of the test setup (i.e. fulfilling the basic requirements as outlined by Stanier \& White (2013)) and subsequent data processing.

Given this best practice in image acquisition conditions (lighting, optical components and camera specifications) and assuming that current PIV algorithms have sufficient accuracy for the purpose, both test cases show similar performance to the state-of-the-art in physical modelling in geomechanics presented by White and co-workers (White et al., 2003) for clay samples. When compared with the work of Dijkstra et al. (2013), slightly better performance was achieved in this study due to a higher speckle density than the natural contrast of large sand grains. The trajectories, consisting of 78 individual points each for one displacement increment, show the curvature in the displacements without any aberration.

\section{CONCLUSIONS}

Speckle patterns can be successfully transferred from a window to clay slurry before subsequent consolidation by preparation of a transparent Perspex window with PTFE spray and the application of lightweight plastic flocks before the clay slurry is poured into a strongbox. This method thus minimises sample disturbance resulting from sample handling and unloading of the sample for removal of the transparent window. Two case studies involving large soil deformations and rotations showed that the performance of the created speckle patterns is at the same level as the state-of-the-art methods used in physical modelling.

\section{REFERENCES}

Allersma, H. G. B. (1994). The University of Delft geotechnical centrifuge. Proceedings of Int. Centrifuge 94 Conf., Singapore, pp. 47-52.

Allersma, H. G. B. (1997). Using imaging technologies in experimental geotechnics. Proceedings of 2 nd Int. Conf. on Imaging Technology Techniques and Civil Engineering Applications, Davos, pp. 1-9.

Badulescu, C., Bornet, M., Dupré, J.-C. et al. (2010). Methodology for metrological analysis of periodic coding images: measurement resolution and spatial resolution. Proceedings of Int. Conf. on Experimental Mechanics, ICEM14, paper 10002. See http://dx.doi.org/10.1051/epjconf/ 20100610002. Accessed 17/03/2014.

Desrues, J. \& Viggiani, G. (2004). Strain localization in sand: an overview of the experimental results obtained in Grenoble using stereophotogrammetry. Int. J. Numer. Anal. Methods Geomech. 28, No. 4, 279-321.

Dijkstra, J., Gaudin, C. \& White, D. J. (2013). Comparison of failure modes below footings on carbonate and silica sands. Int. J. Phys. Model. Geotech. 13, No. 1, 1-12.

Dingle, H. R. C., White, D. J. \& Gaudin, C. (2008). Mechanisms of pipe embedment and lateral breakout on soft clay. Can. Geotech. J. 45, No. 5, 636-652.

Hall, S. A. (2012). Digital image correlation in experimental geomechanics. In ALERT doctoral school 2012: Advanced experimental techniques in geomechanics (Viggiani, G., Hall, S. A. \& Romero, E. (eds)). See http://www.alertgeomaterials. eu/internal/2012/school/2012_ALERT_school.pdf. Accessed $17 / 03 / 2014$

Hall, S. A., Muir Wood, D., Ibraim, E. \& Viggiani, G. (2010). Localised deformation patterning in $2 \mathrm{D}$ granular materials revealed by digital image correlation. Gran. Matter 12, No. 1, $10-14$.

Hossain, M. S. \& Randolph, M. F. (2010). Deep-penetrating spudcan foundations on layered clays: centrifuge tests. Géotechnique 60, No. 3, 157-170.

Leung, C. F., Xie, Y. \& Chow, Y. K. (2008). Use of PIV to investigate spudcan-pile interaction. Proc. 18th Int. Offshore and Polar Engineering Conf., Vancouver, BC, pp. 721-726.

Lunne, T., Berre, T., Andersen, K. H., Strandvik, S. \& Sjursen, M. (2006). Effects of sample disturbance and consolidation procedures on measured shear strength of soft marine Norwegian clays. Can. Geotech. J. 43, No. 7, 726-750.

Meijer, G. J. \& Dijkstra, J. (2013). A novel methodology to regain sensitivity of quick clay in a geotechnical centrifuge. Can. Geotech. J. 50, No. 9, 995-1000

Ni, Q., Hird, C. C. \& Guymer, I. (2010). Physical modelling of pile penetration in clay using transparent soil and particle image velocimetry. Géotechnique 60, No. 2, 121-132.

Ochiai, N., Kraft, E. L. \& Selker, J. S. (2006). Methods for colloid 
transport visualization in pore networks. Water Resources Res. 42, No. 12, 1-14.

O'Loughlin, C. D., Lowmass, A., Gaudin, C. \& Randolph, M. F. (2006). Physical modelling to assess keying characteristics of plate anchors. Proceedings of 6th Int. Conf. on Physical Modelling in Geotechnics, Hong Kong, pp. 659-665.

Rosenbrand, E. \& Dijkstra, J. (2012). Application of image subtraction data to quantify suffusion. Géotech. Lett. 2, No. 2, 37-41.

Stanier, S. A. \& White, D. J. (2013). Improved image-based deformation measurement in the centrifuge environment. Geotech. Test. J. 36, No. 6, 915-928.

Sutton, M., Wolters, W., Peters, W., Ranson, W. \& McNeill, S. (1983). Determination of displacements using an improved digital correlation method. Image Vision Comp. 1, No. 3, 133-139.
Vennemann, P. (2008). JPIV open source software package for particle image velocimetry (PIV). See http://www.jpiv.vennemannonline.de/index.html (accessed 01/08/2013).

White, D. J., Take, W. A., Bolton, M. D. \& Munachen, S. A. (2001). A deformation measurement system for geotechnical testing based on digital imaging, close-range photogrammetry, and PIV image analysis. Proc. 15th Int. Conf. on Soil Mechanics and Geotechnical Engineering, Istanbul, pp. 539542.

White, D. J., Take, W. A. \& Bolton, M. D. (2003). Soil deformation measurement using particle image velocimetry (PIV) and photogrammetry. Géotechnique 53, No. 7, 619-631. Xie, Y. (2009). Centrifuge model study on spudcan-pile interaction. $\mathrm{PhD}$ thesis, National University of Singapore.

\section{WHAT DO YOU THINK?}

To discuss this paper, please email up to 500 words to the editor at journals@ice.org.uk. Your contribution will be forwarded to the author(s) for a reply and, if considered appropriate by the editorial panel, will be published as a discussion. 